\title{
Hacia una perspectiva reticular de la teoría sociológica
}

\author{
Félix Requena Santos
}

Universidad de Santiago de Compostela

frequena@arrakis.es

\section{Resumen}

En este artículo se propone un enfoque de explicación integrada e integradora de la teoría sociológica. Para ello, apoyándonos en el esquema conceptual de Ritzer, se ha construido un modelo que permite ver las diferentes teorías sociológicas desde el punto de vista de la influencia y de las carencias que tienen unas sobre otras. De esta forma, el resultado es una perspectiva reticular de la teoría sociológica. No es un modelo completo, sino un esquema para la incorporación sucesiva de las diferentes teorías o enfoques de la explicación de la realidad social. Es un esquema metateórico que permite el enfoque global de las relaciones que existen entre las diferentes teorías sociológicas.

Palabras clave: enfoque de red, teoría sociológica, metateoría, modelo integral.

Abstract. Towards a reticular or web perspective in socilogical theory

This paper proposes an integral and integrated approach of the sociological theory. Based on Ritzer conceptual scheme, it is constructed a model that shows the influence and lacking between different sociological theories. The result is a network approach of the sociological theory. It is not a complete model, but it is a project for successive addition of different explanation of social reality or social theories. This metatheorical scheme permits a global approach of the relationships between different sociological theories.

Key words: network approach, socillogical theory, metatheory, integral model.

Este artículo permite dar un paso más hacia una explicación integral e integrada de la teoría sociológica, de forma que ayude a explicar las realidades sociales desde los niveles micro y macro sociológicos. La realidad de lo que entendemos por posmodernidad difícilmente se puede entender si no se aúnan ambos niveles de análisis en un esquema integrado.

Una de las cuestiones básicas de la sociología es su diferencia entre objeto material y objeto formal. Existe un relativo acuerdo entre los sociólogos sobre cuál es el objeto material de esta disciplina: las sociedades 
humanas, las colectividades, las relaciones sociales, la realidad empírica de la convivencia como el modo natural de la existencia humana, etc. Sin embargo, hay un gran desacuerdo en cuál es la forma más adecuada de enfrentarse a esa realidad que constituye la convivencia humana, es decir, su objeto formal.

El que un enfoque sea más o menos válido para el acercamiento a la realidad social, parece estar bastante condicionado por el consenso de los sociólogos, consenso que ha variado y continúa variando de unas épocas a otras. Desde este punto de vista, conviene ver a la sociología como una disciplina que es capaz de explicar la realidad social en su complejidad, precisamente debido a los múltiples enfoques que posee. La sociología es una ciencia multiparadigmática, y en estos múltiples enfoques es, precisamente, donde radica su virtud.

Existen muchos y muy variados enfoques sobre la forma de explicar la realidad social. Desde los pioneros de la sociología clásica, tales como el positivismo, el evolucionismo, o la acción social por un lado; o el esquema clásico también de cultura, persona, sociedad y medio ecológico. Por otra parte, desde un esquema más actual, se pueden ver las teorías que tratan de enfocar sobre explicaciones a un nivel más micro u otras a un nivel más macro. Por último, existen también puntos de vista integradores ${ }^{1}$ micro-macro. Dentro de ellos se inserta esta perspectiva reticular.

Los enfoques utilizados en el esquema que se presenta en este ensayo son una muestra representativa. Somos conscientes de que no agotan cada una de las perspectivas, pues el objetivo principal es proponer un esquema explicativo que vea a la teoría sociológica desde una perspectiva reticular. Se trata de mostrar la eficacia de un marco que sea capaz de asumir una de las grandes potencialidades de la teoría sociológica: su multiparadigmaticidad.

Junto con los enfoques clásicos de la dialéctica marxista, los hechos sociales de Durkheim, o la acción social de Weber, entre otros, se ha procurado realizar un recorrido por algunas de las teorías contemporáneas vigentes en la actualidad; desde aquéllas que enfocan a la realidad social desde un punto de vista fundamentalmente microsociológico como la etnometodología o la elección racional, hasta las que lo enfocan desde uno macrosociológico como la teoría crítica o la teoría del sistema mundial, también se han incluido teorías integradoras de estos dos enfoques (micro-macro) de la realidad social, como han sido la teoría de la estructuración o la teoría de redes. Sólo se quiere poner de manifiesto este interés integrador, por ello se ha considerado lo que puede ser un esbozo de interrelación entre las diferentes teorías sociológicas.

1. En el análisis de las teorías sociológicas hay excelentes trabajos globalizadores. Muchos de ellos se comentan a lo largo de este artículo, sin embargo cabe mencionar expresamente los trabajos de Solé, C. «Tendencias actuales en la explicación de la sociedad», Perspecti va Social, 14, Barcelona, 1984; o «Nuevas perspectivas de análisis», en su libro Ensayos de teoría sociológica. Modernización y postmodernidad, Madrid, Paraninfo, 1987, p. 12-29. 
Prácticamente todo el siglo XX se ha caracterizado por un extremismo micro y macro de la teoría sociológica. A partir de la década de los ochenta, comenzando en los Estados Unidos, se inicia un movimiento preocupado por la integración de las teorías micro y macro, y los diferentes niveles de análisis social. Desde estos años, se aprecian por doquier el florecimiento de los trabajos sobre la cuestión del vínculo micro-macro que promete ser un área significativa de avance teórico de la disciplina sociológica ${ }^{2}$. Comienzan a surgir reflexiones interesantes sobre el aspecto multidimensional de la sociología, como son las obras de Alexander ${ }^{3}$, Münch y Smelser ${ }^{4}$, Ritzer ${ }^{5}$, entre otros. Aquí, el esquema reticular que se propone se apoya en los ejes integradores de Ritzer ${ }^{6}$.

Aun concibiendo la importancia integradora de la teoría sociológica, existen dos corrientes principales sobre la integración micro-macro. Los que se centran en la integración de las teorías micro y macro, mientras que otros se preocupan más por el desarrollo de una teoría que analice el vínculo entre los niveles micro-macro. No es lo mismo el intento de integración de las teorías macro (funcionalismo estructural) y micro (el interaccionismo simbólico), que intentar el desarrollo de una teoría que pueda explicar la relación entre los niveles micro y macro. Sin embargo, los esfuerzos más prometedores son aquéllos que intentan integrar teorías sin mostrar una predisposición hacia uno u otro tipo. Probablemente es más interesante la búsqueda de los vínculos que se producen entre las teorías, que situarse en un extremo u otro. Aunque también existe la posibilidad de adoptar un enfoque intrínsecamente integrador que defina lo macro en términos de lo micro y viceversa.

Todo esto nos lleva a lo que ha dado en llamarse la metateorización en sociología, es decir, el estudio profundo de la estructura subyacente a la sociología y sus diversos componentes, entre los que se encuentra la teoría. Es el estudio sistemático de las teorías. Hay tres tipos de metateorización, según cuáles sean los resultados de ésta ${ }^{7}$. Estas tres variedades de metateoría son tipos ideales. En la realidad suele darse un solapamiento considerable de

2. Collins, R.: «Is 1980s sociology in the Doldrums?», American Journal of Sociology, 91, 1986, p. 1336-1355.

3. AleXAnder, J.C. y otros (ed.): The micro-macro link, Berkeley, University of California Press, 1987.

4. MÜnCH, R.; Smelser, N.J.: «Relating the micro and macro». En AleXander, J.C. y otros (ed.): op. cit., p. 356-387.

5. RitZER, G.: «Toward an integrated sociological paradigm». En SNIZEK, W. y otros (ed.): Contemporary issues in theory and research, Westport, Conn., Greenwood Press, 1979, p. 25-46; Toward an integrated sociological paradigm. The search for an exemplar and an image of the subjecct matter, Boston, Allyn y Bacon, 1981.

6. Esta primera parte del ensayo se ajusta al modelo propuesto por Ritzer en su metateoría que establece en Contemporary Sociological Theory, Nueva York, McGraw-Hill, 1992.

7. Ritzer, G.: «Metatheorizing in sociology», Sociological Forum, 5, 1990, p. 3-15; Meta theorizing in sociology, Lexington, Mass., Lexington Books, 1991. 
los objetivos de cada una de ellas, aunque los autores que se encuentran interesados por un tipo suelen no interesarse tanto por los otros dos. Según Ritzer, los tipos de metateorización son los siguientes:

1) La metateorización como medio para obtener una comprensión más profunda de la teoría: es el estudio de la teoría para procurar mejorar la teoría existente, de forma que ayude a comprender mejor una teoría determinada (M1). Este tipo tiene la ventaja de ofrecer métodos sistemáticos para la comprensión, la evaluación, la crítica y la mejora de las teorías existentes.

2) La metateorización como introducción al desarrollo de la teoría: implica el estudio de las teorías existentes para producir una teoría sociológica nueva (M2). La mayoría de los teóricos clásicos y contemporáneos más importantes han desarrollado sus teorías, de forma total o parcial, sobre la base de un estudio meticuloso de la obra de otros teóricos y de su reacción con ella. Ejemplos destacados de ello, han sido la teoría del capitalismo de Marx inspirada en la filosofía hegeliana, en la economía política y en el socialismo utópico; o la teoría de la acción de Parsons desarrollada a partir de un estudio sistemático de la obra de Durkheim, Weber, Pareto, etc. Este tipo de metateorización representa una de las bases más importantes para la creación de una nueva teoría.

3) La metateorización como fuente de las perspectivas que sostienen toda la teoría sociológica: implica un estudio de la teoría enfocado hacia la meta de producir una perspectiva que abarque una parte o toda la teoría sociológica (M3). En este tipo se encuentran todos los trabajos que buscan un paradigma sociológico integrado como el de Ritzer, o una lógica teórica general de la sociología como la de Alexander. Este tipo proporciona a los teóricos perspectivas englobadoras muy útiles para la comprensión general del análisis social.

En general, la gran ventaja que proporciona la metateorización es la creación de teorías nuevas. Éste es el objetivo concreto de la M2, aunque también puede ser el resultado del primer tipo (M1), pues la linea divisoria entre una teoría perfeccionada y una nueva teoría suele ser borroso. Por otro lado, los trabajos en la línea de M3 también pueden conducir a la creación de una teoría nueva. Pero sea cual fuere el caso, la reflexión metateórica sobre la obra de otros teóricos ha sido y sigue siendo una fuente importante para la creación de nuevas teorías, mientras que la tercera función de la metateorización (M3) es la producción de visiones teóricas englobadoras, ya que se centra especialmente hacia la generación de perspectivas totalizantes.

La metateorización también ha recibido críticas. Por ejemplo, Jonathan Turner señala, de forma práctica, que el trabajo metateórico se empantana con frecuencia en cuestiones filosóficas e inmoviliza la construcción teórica, enre- 
dando a los teóricos en controversias intrínsecamente insuperables y siempre discutibles $^{8}$. O la de Randall Collins, que ve la metateorización como una especialidad reflexiva, que sólo hace comentarios sobre otros campos a los que se refieren otras obras del pasado, en lugar de realizar construcciones creativas por sí mismas?.

\section{II}

Una perspectiva multiparadigmática. Apoyándonos en el concepto de paradigma de Kuhn ${ }^{10}$, se puede ver a la sociología como una ciencia multiparadigmática. Son varios los que han visto a la sociología de esta forma, por ejemplo, Friedrichs ${ }^{11}$ presentó dos imágenes distintas del estatus paradigmático de la sociología, sin embargo, ambas enfocan hacia la idea de que la sociología es una disciplina multiparadigmática. Por un lado, la sociología se ha dividido entre el paradigma sistémico, que acentúa la integración societal y el consenso, y un paradigma del conflicto, que acentúa la desintegración societal y la coerción.

Otro ejemplo sustancial es el de Eisenstadt y Curelaru ${ }^{12}$, que diferencian entre el paradigma discreto, el del sistema cerrado y el del sistema abierto. El paradigma discreto se centra en entidades concretas separadas, tales como las propiedades ecológicas, el tamaño de los grupos o las características raciales, que proporcionan una imagen del mundo como un conjunto de unidades aisladas; este paradigma tiene dificultades a la hora de analizar cuestiones relacionales tales como la innovación o la creatividad. El paradigma del sistema cerrado, que sustituyó con el tiempo al paradigma anterior, cree que un elemento domina sobre los demás, así, por ejemplo, Marx usó este paradigma de forma que enfatizaba en el sector económico. Una vez más, este paradigma fue reemplazado por el del sistema abierto, que se centra en la dinámica interna del sistema, las interconexiones y los procesos de interacción continua entre los componentes del orden social.

Por último, otro ejemplo interesante de esta visión multiparadigmática es la del propio Ritzer ${ }^{13}$, que observa la existencia de tres grandes paradigmas que dominan la sociología, y que existen junto con otros que potencialmente pueden alcanzar el estatus de paradigma. Estos tres paradigmas principales son el de los hechos sociales, el de la definición social y el de la

8. TURner, J.: The structure of sociological theory, Chicago, Dorsey Press, 1986, p. 10.

9. COllins, R.: op. cit.

10. KIHN, T.S.: La estructura de las revoluciones cientificas, Madrid, Fondo de Cultura Económica, 1977.

11. Friedrichs, R.: Sociología de la sociología, Buenos Aires, Amorrortu, 1977.

12. Eisenstadt, S.N.; Curelaru, M.: The form of sociology: paradigms and crises, Nueva York, Wiley, 1976, p. 92.

13. Ritzer, G.: Sociology: A multiple paradigm science, Boston, Allyn y Bacon, 1980. 
conducta social. El de los hechos sociales está marcado en la obra de Durkheim, sus partidarios se centran en las grandes instituciones y estructuras sociales y en su influencia sobre el pensamiento y la acción individuales; fruto de este paradigma son las teorías del funcionalismo estructural, la del conflicto o la teoría de sistemas. El paradigma de la definición social, en cambio, se apoya en la obra de Weber sobre la acción social, que suscita interés por la forma cómo los actores definen sus situaciones sociales y la influencia de estas situaciones en la acción e interacción consecuentes; de este paradigma surgen la teoría de la acción, el interaccionismo simbólico, la fenomenología o la etnometodología. Por último, el paradigma de la conducta social se sustenta en la obra de Skinner, donde se apoyan los conductistas sociales que ven a la conducta como fruto de recompensas y castigos; resultado de éste son por ejemplo la sociología conductista o la teoría del intercambio.

Estas aproximaciones multiparadigmáticas de la sociología demuestran una gran utilidad, sin embargo también es necesaria la creación de un paradigma más integrado y, sobre todo, integrador. Los paradigmas que existen, aunque complementarios, tienden a ser parciales con lo que enfatizan niveles específicos del análisis social, sin considerar demasiado lo que hacen los otros paradigmas ${ }^{14}$.

La realidad social consta de una enorme complejidad de fenómenos sociales que experimentan una continua interacción y un cambio constante. La gran multitud de instituciones, grupos y procesos que constituye la realidad social resulta muy difícil de controlarse de forma conjunta si no se observa desde un esquema conceptual propio para tal finalidad. Para ello, nos apoyaremos en el esquema conceptual de Ritzer ${ }^{15}$ que establece dos ejes de la realidad social, para explicar el desarrollo de los principales niveles del mundo social.

Por un lado, un eje micro-macro en función de la magnitud y la escala con la que se traten los fenómenos sociales. Así, por ejemplo, en el extremo macro se sitúan los sistemas mundiales, las sociedades, las culturas, etc.; mientras que en el extremo micro de este eje se sitúan los actores individuales, sus pensamientos y sus acciones. Entre ambos extremos, en un nivel intermedio del eje, se sitúan un amplio conjunto formado por grupos, colectividades, organizaciones o clases sociales, etc.; aunque, por supuesto, no hay una línea divisoria clara entre las unidades macrosociales y las microsociales.

Por el otro lado, el eje referido a la dimensión objetivo-subjetivo del análisis social. Lo objetivo hace referencia a los hechos reales y materiales que ocurren en la vida real, son fácilmente observables. Son los actores y su acción, la interacción, las estructuras burocráticas, el aparato del Estado, etc.

14 Ibídem.

15. Ritzer, G.: «Toward an integrated sociological paradigm...», op. cit. 
Mientras que lo subjetivo se refiere a lo que ocurre exclusivamente en el campo de las ideas, y que carecen de una existencia material, son los procesos mentales, la construcción social de la realidad, las normas, los valores y otros muchos elementos de la cultura. Este eje tiene una dificultad importante que reside en que hay muchos fenómenos que contienen tanto elementos objetivos como subjetivos. Por ejemplo, el trabajo se compone de una existencia material, como las tareas y actividades que hay que realizar en el trabajo, y de una serie de normas y valores e ideales respecto al trabajo.

Cruzando ambos ejes nos encontramos con un plano dividido en cuatro cuadrantes según del esquema de Ritzer ${ }^{16}$ : 1) macro-objetivo, por ejemplo la sociedad, el derecho, la burocracia, etc.; 2) macro-subjetivo, formado entre otros elementos por la cultura, las normas, los valores, etc.; 3) micro-objetivo, son entre otros las pautas de conducta, acción e interacción, etc., y 4) micro-subjetivo, que comprende el pensamiento, definición personal de la situación, percepción, etc. Estos cuadrantes son los que usaremos como marco de referencia para insertar nuestra perspectiva reticular.

\section{III}

Hacia una visión reticular de la teoría sociológica. El objetivo es trazar una propuesta de red que integre las diferentes teorías a través de las relaciones de influencia y/o carencia que muestran entre sí tales teorías. En este primer esbozo se establece una red con una muestra representativa de diversas teorías. Es evidente que se trata, todavía, de una red incompleta, pero a medida que se vaya observando su utilidad como perspectiva integradora de la teoría sociológica, se deberá ir ampliando en el futuro.

Las diferentes teorías se han colocado en los cuatro cuadrantes propuestos por Ritzer, en función de los dos ejes: micro-macro y subjetivo-objetivo. Cada teoría está posicionada de forma que su objeto coincida con los criterios reseñados para cada una de las divisiones efectuadas en el plano establecido por tales ejes. Así, por ejemplo, los cuatro extremos del plano, que coinciden con la formulación más rigurosa de los cuatro extremos de los ejes, podrían ser los siguientes: En primer lugar, el enfoque dialéctico de Marx que mira a la sociedad como una gran estructura donde las relaciones principales están determinadas económicamente, se encuentra en el extremo más macro y objetivo del plano de clasificación. En segundo lugar, la teoría crítica, que realiza un conjunto de reflexiones sobre la incidencia del sistema capitalista en la moral y en las conciencias de los miembros sociales, se encuentra en uno de los extremos macro y subjetivo. En tercer lugar, en el extremo micro-objetivo se encuentra la etnometodología; esta teoría enfoca

16. Ibíd. 
hacia las interacciones concretas de los individuos en las situaciones reales de la vida cotidiana. Y por último, la fenomenología se encuentra en el mismo plano micro, pero en el extremo subjetivo, ya que su objeto es individual, pero hace referencia al mundo de los símbolos y las ideas (figura 1).

La figura 1 muestra una malla en la que se observan los diferentes enfoques teóricos y sus relaciones. Las relaciones están marcadas con un signo positivo (+) o negativo (-), de forma que las flechas con signo (+) indican procesos de influencia entre dos enfoques teóricos, mientras que las flechas con signo (-) indican las carencias de unas teorías sobre otras materializadas con lagunas explicativas que argumentan las teorías de las que parte la flecha. Este esquema reticular proporciona una perspectiva conjunta, tanto de las proximidades teóricas entre dos o más enfoques como las necesidades de un enfoque particular de profundizar en aspectos determinados de los que carece. Este modelo proporciona una herramienta metateórica de utilidad para el análisis comparado de los teóricos de la sociología; de forma que ayuda a analizar las relaciones y proporciona una base relacional para el análisis de los diversos enfoques teóricos de la sociología.

A continuación desarrollamos las relaciones implicadas en la figura 1 siguiendo el criterio secuencial siguiente: desde el extremo macro-objetivo, siguiendo por el macro-subjetivo y el micro-objetivo, hasta llegar al microsubjetivo; aunque ésta sea la secuencia preferencial es evidente que algunas relaciones implicarán retrocesos o avances entre los diferentes cuadrantes cuando nos refiramos a relaciones concretas entre dos enfoques teóricos.

En el primer cuadrante macro-objetivo, en primer lugar nos encontramos con el enfoque marxista que influye (relación +) en nuestra red a la teoría del sistema mundial de Wallerstein a través del determinismo económico. Asimismo, el enfoque marxista influye $(+)$, a través de la visión dialéctica, en la teoría del conflicto situada en este mismo cuadrante, aunque más cerca del nivel micro. También, el enfoque marxista influye (+), a través de la dialéctica, en la teoría crítica, ya situada en el cuadrante macro-subjetivo.

El sistema mundial, influído (+) por el enfoque marxista a través del determinismo económico en su visión de la historia; y sin embargo, presenta las carencias (-) de una explicación del factor ideológico y cultural en el sentido de Weber, ya que suprime la explicación que tiene, por ejemplo la religión en el desarrollo mundial.

El enfoque del hecho social de Durkheim influye (+) en la teoría crítica a través de la visión de que las ideas son hechos sociales, dentro ya del nivel subjetivo. También influye $(+)$ en la teoría de la estructuración a través de la importancia de los hechos sociales, y tiene también un sentido $(-)$ por la negación de la estructura como algo exterior o externa a la acción humana, sino que está creada por la acción de los agentes. Por otra parte, influye (+) en la etnometodología a través de que lo fundamental sean siempre los hechos sociales. También se aprecia una carencia (-) importante de los hechos sociales en la teoría del intercambio. Este enfoque es, básicamente, un reacción al paradigma holista. 


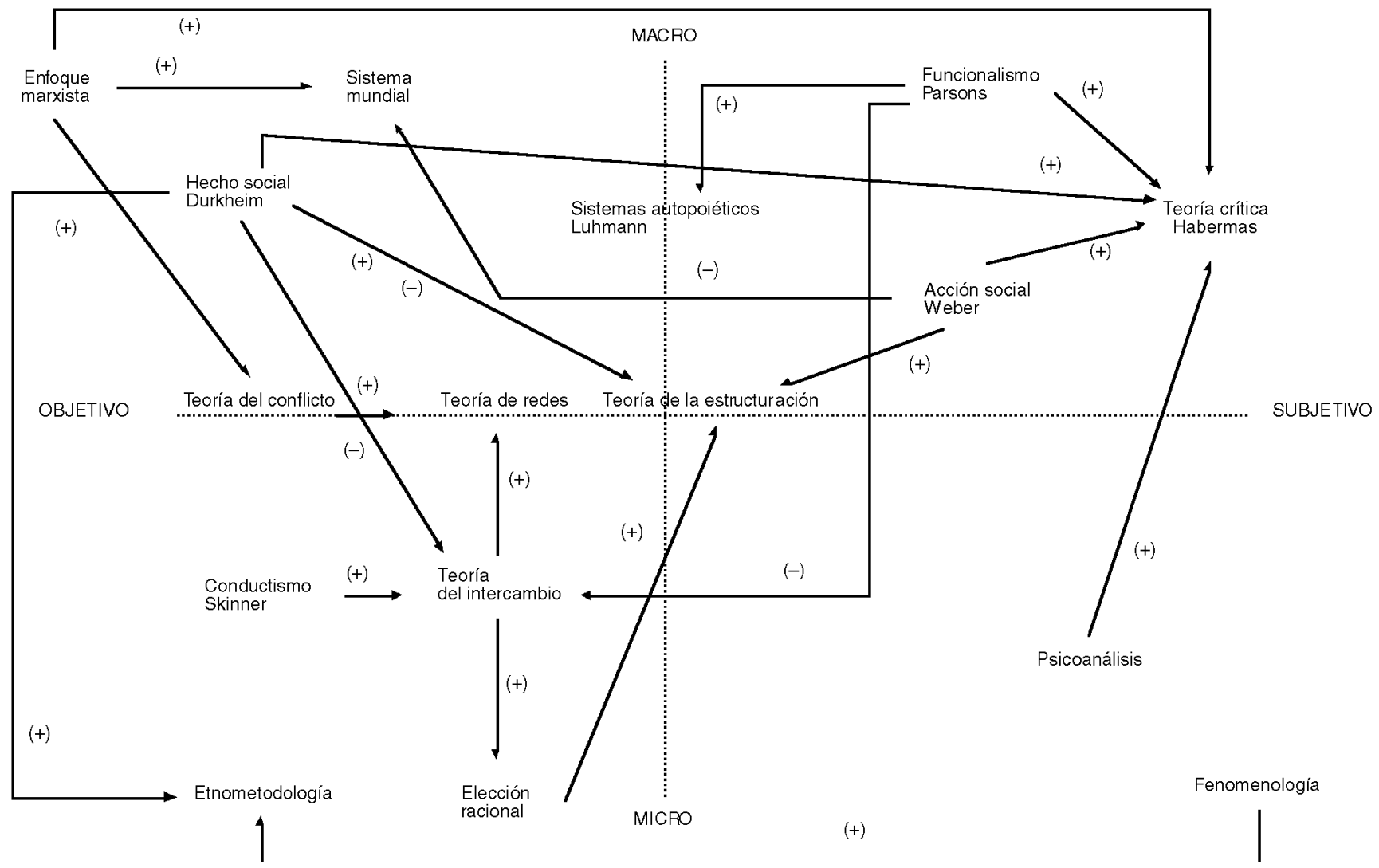

Figura 1. Perspectiva reticular de la teoría sociológica. 
En el límite entre lo subjetivo y lo objetivo, dentro del nivel macro, se sitúa la teoría de los sistemas autopoiéticos de Luhmann, que se ve influido (+) por la visión sistémica parsoniana.

En el cuadrante macro-subjetivo, el funcionalismo parsoniano influye (+) a través de los sistemas de acción y la visión sistémica en la teoría crítica y, en concreto, en la acción comunicativa de Habermas.

La teoría crítica, más que influir sobre algunos, influyen (+) sobre ella distintos enfoques como los siguientes: el enfoque marxista a través de la dialéctica, el hecho social durkheimiano a través de la concepción de las ideas como hechos sociales, y la visión parsoniana a través de los sistemas de acción y la visión sistémica, antes referidos; la teoría weberiana le influye (+) a través de la concepción de los tipos ideales y de la racionalización, fundamentalmente en Habermas y, por último, la teoría crítica es afectada (+) por los argumentos psicoanalíticos procedentes del psicoanálisis freudiano.

La acción social de Weber influye (+) en la acción comunicativa de Habermas de la teoría crítica como ya se ha visto, e influye $(+)$ en la teoría de la estructuración a través de la idea de intencionalidad, los actores tienen motivaciones para actuar y estas motivaciones implican deseos que impulsan la acción, así la racionalidad y la reflexividad están constantemente implicadas en la acción, lo que implica una clara intencionalidad en la acción.

En el límite micro-macro del extremo objetivo, la teoría del conflicto influye $(+)$ en la teoría de redes a través del conflicto/poder entre los actores, ya sean éstos individuales o colectivos, a su vez se ve influida $(+)$ por la visión dialéctica del enfoque marxista, que anteriormente ha sido referido.

La teoría de redes, también en el límite micro-macro, se ve influida (+) por la teoría del intercambio, tomando de ella, en sus versiones más elaboradas de Emerson y Cook ${ }^{17}$, las interpretaciones interactivas entre actores; también está influida $(+)$ por la teoría del conflicto, a través de las relaciones conflictivas entre los actores.

El conductismo influye $(+)$ a través de los experimentos de Skinner sobre las implicaciones que conllevan para la acción las recompensas/castigos en acciones similares anteriores, en la teoría del intercambio de Homans.

La teoría del intercambio influye (+) sobre la teoría de redes ya mencionada, e influye $(+)$ sobre la teoría de la elección racional. Se ve influida $(+)$ por los experimentos conductistas de Skinner. Sin embargo, presenta las carencias (-) de no prestar atención a las coerciones externas durkheimianas, y también carece (-) de atención hacia las instituciones del argumento parsoniano.

17. Tanto Emerson como su colega Karen Cook elaboran un esquema, más flexible que el de Homans, de la teoría del intercambio, centrada en las redes de intercambio donde las posiciones estructurales y la centralidad en el poder desarrollan papeles importantes. Véase CoOK, K.S.; Emerson, R.M.; Gillmore, M.B.; Yamagishi, T.: «The distribution of power in exchange networks: theory and experimental results", American Journal of Socio logy, 89, 1983, p. 275-305; y EMERSON, R.M.: "Social exchange theory», en RosENBERG, M.; TURner, R.H. (ed.): Social Psychology: sociological perspectives, Nueva York, Basic Books, 1981, p. 30-65. 
La elección racional está influida (+) por la teoría del intercambio a través de la concepción de Homans sobre interés coste/beneficio de las acciones. La elección racional, por otra parte, influye $(+)$ sobre la teoría de la estructuración en la idea de que los individuos son conocedores del mundo en que viven y actúan, y las posibles consecuencias y efectos de su acción.

En el extremo más micro de este cuadrante micro-objetivo, se encuentra la etnometodología, que está influida $(+)$ por la teoría del hecho social de Durkheim, como ya se señaló, e influida $(+)$ por la fenomenología a través de una clara influencia de la obra de Schutz, que supone un interés común por el modo en que las personas producen activamente y mantienen los significados de las situaciones de la vida cotidiana.

En el cuadrante micro-subjetivo, se pueden situar el psicoanálisis, que influye $(+)$ a través de los argumentos psicoanalíticos en la teoría crítica; y la fenomenología que, como hemos visto antes, marca una gran influencia $(+)$ en la etnometodología de Garfinkel.

En una posición central, en la intersección de ambos ejes, se ha situado la teoría de la estructuración. Esta teoría ocupa esta posición debido a que es uno de los esfuerzos más conocidos y esmerados por integrar la acción y la estructura, lo que conlleva una posición intermedia entre las grandes estructuras macro y la acción individual micro; enfoca hacia las prácticas sociales que integran la visión dual de acción y estructura, ya que toda acción implica una estructura, y toda estructura afecta a la acción social. Acción y estructura serían como las dos caras de una misma realidad, y se encuentran inextricablemente unidas en toda actividad humana. Esta teoría está influida $(+)$ por la teoría de la acción social de Weber a través de la idea de la intencionalidad, influida $(+)$ por la elección racional a través de una visión individual de la acción; y por último, está influida $(+)$ también por la teoría del hecho social de Durkheim, debido a la importancia que se le presta a éstos en esta teoría, sin embargo tiene la carencia (-) de considerar a la estructura como algo no exterior, sino creada por los propios agentes.

Este enfoque reticular tiene la utilidad añadida de ver, no sólo los orígenes directos de una teoría, sino que es posible siguiendo la trayectoria secuencial de las relaciones de las teorías que le afectan, ver los orígenes indirectos en los que se apoya una teoría determinada o, por el contrario, percibir cuáles son las carencias globales que se le pueden atribuir a tal teoría.

A pesar de la claridad de esta visión global de la teoría sociológica, hay que recordar que en el mundo real lo que existe es un gran continuo social, más que unos ejes micros y macros u objetivos y subjetivos. Por tanto, lo que se ha construido son más bien diferenciaciones arbitrarias para poder analizar la realidad social, sin embargo el interés heurístico de esta argumentación reticular es más que evidente, aunque no aspira a ser una descripción detallada del mundo social.

En este pequeño ensayo se ha descrito un modelo, partiendo del esquema de Ritzer, para enfatizar la imagen del objeto de un paradigma sociológico integrado centrado en la gran red de la teoría sociológica. No conviene 
olvidar que este modelo de interpretación es sólo una muestra y un proyecto de organización de las diferentes teorías sociológicas. Modelo que permite la inclusión sucesiva y ulterior del resto de las teorías. Sin embargo, el modelo no ha pretendido en ningún momento ser exhaustivo, sino simplemente explicativo respecto a una forma de integrar y relacionar las teorías sociológicas. Este esquema requiere una mayor agregación que se logrará con profundizaciones ulteriores. Sin embargo, no hay que olvidar que no se trata de desarrollar un paradigma sociológico nuevo, sino mostrar un esquema metateórico que permita una perspectiva totalizante de las relaciones por las que se influyen y son influidas las diferentes teorías sociológicas.

\section{Referencias bibliográficas}

AleXANDER, J.C. y otros (ed.) (1987). The micro-macro link. Berkeley: University of California Press.

Collins, R. (1986). "Is 1980s sociology in the Doldrums?». American Journal of Sociology, 91: 1336-1355.

CoOK, K.S.; Emerson, R.M.; Gillmore, M.B.; Yamagishi, T. (1983). «The distribution of power in exchange networks: theory and experimental results». Ame rican Journal of Sociology, 89: 275-305.

EISENSTADT, S.N.; CURELARU, M. (1976). The form of sociology: paradigms and cri ses. Nueva York: Wiley.

EMERSON, R.M. (1981). «Social exchange theory». En RosenBerG, M.; TuRner, R.H. (ed.). Social Psychology: sociological perspectives. Nueva York: Basic Books, p. 30-65.

FrIEDRICHS, R. (1977). Sociología de la sociología. Buenos Aires: Amorrortu.

Kunn, T.S. (1977). La estructura de las revoluciones científicas. Madrid: Fondo de Cultura Económica.

MÜNCH, R.; SMElSER, N.J. «Relating the micro and macro». En AleXANDer, J.C. y otros (ed.), op. cit., p. 356-387.

Ritzer, G. (1992). Contemporary Sociological Theory. Nueva York: McGraw-Hill.

- (1990). «Metatheorizing in sociology». Sociological Forum, 5, p. 3-15.

- (1991). Metatheorizing in sociology. Lexington, Mass.: Lexington Books.

- (1979). "Toward an integrated sociological paradigm». En SNIZEK, W. y otros (ed.). Contemporary issues in theory and research. Westport, Conn.: Greenwood Press, p. 25-46.

- (1980). Sociology: A multiple paradigm science. Boston: Allyn y Bacon.

- (1981). Toward an integrated sociological paradigm. The search for an exemplar and an image of the subjecct matter. Boston: Allyn y Bacon.

SOlÉ, C. (1987). «Nuevas perspectivas de análisis». En: Ensayos de teoría sociológica. Modernización y postmodernidad. Madrid: Paraninfo, p. 12-29.

- (1984). «Tendencias actuales en la explicación de la sociedad». Perspectiva Social, 14. Barcelona.

Turner, J. (1986). The structure of sociological theory. Chicago: Dorsey Press. 